\title{
Un modelo para el desarrollo del Pensamiento
} Histórico

\section{A model for the development of historical thinking}

\section{Chávez Preisler, Carolina}

\section{Carolina Chávez Preisler}

carolina.chavez@pucv.cl

Pontificia Universidad Católica de Valparaíso, Chile

Clio \& Asociados. La historia enseñada

Universidad Nacional del Litoral, Argentina

ISSN: 2362-3063

Periodicidad: Semestral

núm. 33, 2021

clio@fhuc.unl.edu.ar

Recepción: 07 Junio 2021

Aprobación: 18 Octubre 2021

URL: https://doi.org/10.14409/cya.v0i33.10355

\section{c) (i) (2)}

Esta obra está bajo una Licencia Creative Commons AtribuciónNoComercial-CompartirIgual 4.0 Internacional.
Resumen: El objetivo del artículo es sistematizar un modelo para el desarrollo del Pensamiento Histórico. El modelo es el resultado de una investigación doctoral desarrollada desde la Universidad Autónoma de Barcelona, en la que se indaga el desarrollo del Pensamiento Histórico en Formación Inicial Docente en seis universidades chilenas (Chávez, 2020). La metodología utilizada es cualitativa. El modelo emerge desde la interacción entre el análisis de los datos (narrativas y observación en universidades) y la revisión bibliográfica. Las particularidades de la propuesta respecto a sus precedentes (Duquette, 2011; Hassani, 2005; Martineau, 1999; Santisteban, 2010; Seixas y Morton, 2013) son: que ha sido pensada para promover el desarrollo del Pensamiento Histórico desde la enseñanza; emerge desde la interacción entre la teoría y la práctica; y en su estructura incluye dimensiones, componentes e indicadores cuya explicitación facilita la enseñanza de la disciplina.

Palabras clave: pensamiento histórico, formación inicial docente, didáctica de la historia, enseñanza de la historia, modelo de pensamiento histórico.

Abstract: The aim of this papers is to organise a model for further development of the Historical Thinking. This organization is the outcome of a previous PhD study carried out at the Autonomous University of Barcelona, which enquired into the development of Historical Thinking in initial teacher training in six Universities (Chávez 2020). The method used to acquire data was qualitative. The data comes from the interaction between participants'persectives and observation at universities, as well as literature review. The distinctive features of this proposal from previous studies (Duquette, 2011; Hassani, 2005; Martineau, 1999; Santisteban, 2010; Seixas y Morton, 2013) are: It has been thought to promote Historical Thinking from a teaching context, which is based on the interaction between theory and practice. Besides, its structure includes dimensions, components and indicators which aids to teaching the discipline.

Keywords: historical thinking, initial teacher training, history didactics, history teaching, model of historical thinking. 


\section{Introducción}

El Pensamiento Histórico es objeto de interés de diversas disciplinas: la historia, lahistoriografía, la filosofía de la historia y la teoría de la historia. Se valoran los aportes provenientes de ellas, pues permiten reconocer y teorizar sobre los elementos y procedimientos que intervienen en la construcción de la disciplina. No obstante, han sido la psicología y la didáctica las disciplinas que han vinculado el Pensamiento Histórico con la enseñanza y el aprendizaje y han permitido, además, desarrollar modelos conceptuales para la comprensión pedagógica del lenguaje historiográfico, por ejemplo: Duquette (2011); Hassani (2005); Lautier (1994); Martineau (1999); Santisteban (2010); Seixas y Morton (2013) o van Drie y van Boxtel (2008).

Se entiende por modelo de Pensamiento Histórico una sistematización, organización y conceptualización de componentes o procedimientos propios de la historia (Duquette, 2011) que han sido considerados por historiadores/as para hacer y reconstruir la disciplina. Martineau (1999) señala que el pensamiento funciona sobre la base de una red de procedimientos y componentes, esto explica la importancia de generar modelos para comprender y desarrollar el Pensamiento Histórico.

En la enseñanza de la historia, la didáctica ha puesto en evidencia las dificultades que presenta el profesorado para desarrollar el Pensamiento Histórico: Belanger (2011); Chávez y Pagès (2019, 2020); Chávez (2020); Gibson y Peck (2020); Hassani (2005); Martineau (1999); Ramírez Achoy y Pagès (2019). Para abordar y contribuir a aplacar estas dificultades se propone un modelo pensado para favorecer la enseñanza de la disciplina. La necesidad de sistematizarlo se justifica porque como proponen Chaparro et al., (2020), es importante que emerjan nuevas propuestas en las que se incorporen la perspectiva de género, el enfoque decolonial y la interdisciplinariedad. Se piensa que el modelo de Pensamiento Histórico que se expone en los resultados de este artículo responde a estas necesidades.

\section{Marco Teórico}

\subsection{Pensamiento Histórico}

El Pensamiento Histórico es un proceso articulado por operaciones propias de la historia y cuyo objetivo es responder a un problema específico a través de la interpretación de las fuentes (Duquette, 2011). Además posee particularidades que permiten ampliar la comprensión de lo que es e implica pensar históricamente Chávez (2020) expone que el Pensamiento Histórico:

Es contextual, es decir, cada cultura comprende la temporalidad y el espacio particularmente (Seixas y Morton, 2013) y en su comprensión intervienen elementos como la identidad, la memoria, la experiencia y las emociones (Carretero, 2007).

Es articulado y jerarquizable, esto significa que las dimensiones y los componentes propios de la historia no se desencadenan aisladamente (Bermejo, 1991; y Seixas y Morton, 2013), al contrario, se engranan (Ricoeur, 2000) 
simultáneamente (Carretero y Castorina, 2012), donde la realidad es la síntesis y la coordinación de diversos elementos como el tiempo, el espacio, la causalidad u otros (Bermejo, 1991). En particular para la enseñanza de la historia, las diferentes dimensiones y componentes que configuran el Pensamiento Histórico adquieren una jerarquía dependiendo de las necesidades de análisis o interpretación (van Drie y van Boxtel, 2008). La jerarquización ha de estar en coherencia con los objetivos de aprendizaje y con el contenido histórico, esto quiere decir que habrá ocasiones en que se priorice el desarrollo de algunas dimensiones y/o componentes del Pensamiento Histórico por sobre otras.

Es preferentemente hipotético deductivo porque la historia considera la problematización, el planteamiento de preguntas y supuestos, el uso de fuentes y la emisión de explicaciones argumentadas (Duquette, 2011; Gómez et al., 2014; Hassani, 2005), elementos que son vectores del Pensamiento Histórico (Martineau, 1999).

Es reflexivo, crítico e interpretativo esto quiere decir que permite navegar en la relatividad y los puntos antagónicos como lejano/cercano o familiar/ajeno (Carretero y Kriger, 2012) y facilita la comprensión del acontecer histórico (Stearns, 2004) desarrollando una visión crítica y argumentada de la realidad (Martineau, 1999; Hassani, 2005; Lévesque, 2008).

Es único porque es una construcción que emerge de cada ser histórico y que está influenciada por su sistema de creencias (Bermejo, 1991). Esto repercute, por ejemplo, en que cada persona frente a un mismo acontecer tendrá sus propias interpretaciones (Collingwood, 1952).

Es antinatural, esto se explica porque es más sencillo recordar nombres, fechas y relatos que pensar históricamente el pasado, articulando y subsumiendo (sintetizando y relacionando) los diferentes componentes de la historia (Bermejo, 1991; Wineburg, 1991 y 2001).

Es progresivo porque cada persona posee, dependiendo de la edad, formación y/o contexto, diferentes niveles de desarrollo de las operaciones propias de la historia (Carretero y Kriger, 2012).

\subsection{Modelos de Pensamiento Histórico}

Existen diversas propuestas para el desarrollo del Pensamiento Histórico. Las diferencias entre ellas son una consecuencia de las dimensiones, los conceptos estructurantes o componentes y las habilidades cognitivas y/o procedimentales que involucren. Las características de estos modelos nos permiten afirmar que: i) hay un predominio de las investigaciones anglófonas y francófonas (Duquette, 2011; Hassani, 2005; Martineau, 1999; Seixas y Morton, 2013; Seixas y Peck, 2004; van Drie y van Boxtel, 2008) en desmedro de las hispanoparlantes (Santisteban, 2010); ii) existen antecedentes de modelos que combinan la teoría y los datos que aporta el trabajo de campo (Duquette, 2011; Hassani, 2005 y Martineau, 1999); y iii) el modelo más acabado es el de Seixas y Morton (2013) pero, por sí solo y para esta investigación, presenta limitaciones. Para la elaboración del modelo de Pensamiento Histórico que se presenta en este artículo se consideran los aportes de:

Duquette (2011), pues la interpretación de este modelo permitió i) distinguir que en un modelo de Pensamiento Histórico hay dimensiones y procedimientos 
propios de la historia; e ii) identificar las dimensiones cognitiva, metodológica y social.

Santisteban (2010) y Santisteban et al. (2010): de este modelo se incluyen los elementos de la conciencia histórica-temporal, la empatía histórica y la representación histórica.

Seixas y Peck (2004), Seixas (2006) y Seixas y Morton (2013), los aportes que se consideran son: los seis conceptos de Pensamiento Histórico y algunos de sus indicadores.

Martineau (1999), Hassani (2005) y van Drie y van Boxtel (2008) respecto a estos modelos se reconocen sus diferencias individuales, no obstante, se extrae de ellos un denominador común que no está presente explícitamente en los otros modelos descritos. Esto corresponde a la problematización y la pregunta histórica.

\section{Metodología}

El objetivo del artículo consiste en sistematizar un modelo para el desarrollo del Pensamiento Histórico. La investigación se posiciona desde la teoría y el paradigma socio-crítico porque contribuye a la transformación sociohistórica (Freire, 2004). Este paradigma es importante para interpretar, comprender y analizar la enseñanza de las ciencias sociales y la historia (Pagès y Santisteban, 2011).

La metodología utilizada es cualitativa ya que permite comprender relaciones (Flick, 2015) y favorecer el refinamiento y la extensión de teorías (Sandoval, 1996), a través de la interacción constante entre referencias teóricas, metodologías y la recogida de información (Rojas, 2015). La sistematización del modelo se lleva a cabo articulando la teoría y los datos (Arnal et al., 1992). La teoría proporciona un marco referencial inicial para interpretar la información del trabajo de campo (Maxwell, 2005).

\subsection{Muestra}

La muestra la constituyen 69 estudiantes de formación inicial pertenecientes a cinco universidades de Chile (Universidad de Santiago de Chile, Universidad Metropolitana de las Ciencias de la Educación, Universidad, Universidad de Valparaíso, Universidad de la Frontera y Universidad Austral de Chile) y una docente de didáctica, doctora en educación con especialidad en didáctica.

2.2. Procedimientos de recogida y análisis de información Para la sistematización del modelo de enseñanza del Pensamiento Histórico se levantó información proveniente de investigaciones historiográficas y didácticas, narrativas de 69 estudiantes de Formación Inicial y observación de tres clases de didáctica.

- Investigaciones historiográficas y didácticas: a través de estas fuentes de información se identificaron los diferentes modelos de Pensamiento Histórico y se describieron dimensiones y componentes. 
- Narrativa: es un texto organizado que permite ordenar las ideas interrelacionadamente a través de un relato profundo (Reis y Climent, 2012). Es la forma lingüística que sintetiza la temporalidad y da sentido al pasado (Rüsen, 2004), re-describe lo que alguna vez ocurrió a través de una interacción entre la experiencia individual y colectiva (Carr, 2015). En didáctica, las investigaciones de Sáiz y Gómez (2016) y Sant et al. (2014) utilizan las narrativas del estudiantado para indagar sobre el Pensamiento Histórico y han sido consideradas en esta investigación. Las instrucciones para desarrollar la narrativa, fueron: i) elija libremente un contenido histórico del currículum y relate su enseñanza, ii) de ser necesario puede consultar internet cuando estime conveniente.

- Observación: es un proceso sistemático que permite indagar cómo se desenvuelve un fenómeno en su contexto real (Ruíz, 2012). Se desarrolló observación no participante (Bisquerra, 2009) en tres clases (7,3 horas) de didáctica de la historia y en una universidad.

- El análisis de la información se ejecutó a través de análisis de contenido y categorial siguiendo las indicaciones de Bardin (2002) y Ruíz (2012). La información ha sido procesada con el software estadístico Atlas-ti, 8.0.

\section{Resultados}

\subsection{Fases de elaboración del modelo}

La elaboración del modelo se llevó a cabo en cuatro fases, en las que se incluye la revisión y el análisis teórico, el análisis de narrativas, la observación de clases de didáctica y la redefinición del modelo inicial.

- Primera fase: Consistió en analizar la información procedente de 25 narrativas utilizando las categorías teóricas de Santisteban (2010) y Seixas y Morton (2013). Las categorías, utilizadas, se sintetizan en la Tabla 1. 
Tabla 1.

Categorías iniciales.

\begin{tabular}{|l|l|l|}
\hline Categoría & Referentes & Componentes \\
\hline $\begin{array}{l}\text { Consciencia } \\
\text { temporal-histórica/ cambio y } \\
\text { continuidad }\end{array}$ & Santisteban (2010), & $\begin{array}{l}\text { Pasado, presente, futuro, operadores } \\
\text { temporales y tiempo histórico. }\end{array}$ \\
\cline { 2 - 3 } & $\begin{array}{l}\text { Seixas y Morton } \\
(2013)\end{array}$ & Cambio y continuidad \\
\hline Perspectiva histórica/ empatía & Santisteban (2010), & Empatía histórica \\
\cline { 2 - 3 } & $\begin{array}{l}\text { Seixas y Morton } \\
(2013)\end{array}$ & $\begin{array}{l}\text { Perspectiva histórica, } \\
\text { contextualización, cambio }\end{array}$ \\
\hline Representación & Santisteban (2010) & $\begin{array}{l}\text { Hechos, procesos, protagonistas, } \\
\text { espacio. }\end{array}$ \\
\hline Causas y consecuencias & $\begin{array}{l}\text { Santisteban (2010), } \\
\text { Seixas y Morton } \\
(2013)\end{array}$ & $\begin{array}{l}\text { Causas, consecuencias, } \\
\text { multicausalidad, jerarquia. }\end{array}$ \\
\hline Significancia histórica & $\begin{array}{l}\text { Seixas y Morton } \\
(2013)\end{array}$ & $\begin{array}{l}\text { Continuidad, consecuencias, cambio, } \\
\text { ámbitos. }\end{array}$ \\
\hline $\begin{array}{l}\text { Interpretación histórica y uso } \\
\text { de evidencia }\end{array}$ & $\begin{array}{l}\text { Santisteban (2010), } \\
\text { Seixas y Morton } \\
(2013)\end{array}$ & $\begin{array}{l}\text { Uso de fuentes y /o evidencias, } \\
\text { (contextualización, análisis y } \\
\text { contrastación) }\end{array}$ \\
\hline Dimensión ética & $\begin{array}{l}\text { Santisteban (2010), } \\
\text { Seixas y Morton } \\
(2013)\end{array}$ & Juicio ético/ moral Valores \\
\hline
\end{tabular}

Estas categorías y sus respectivos componentes fueron útiles para analizar las narrativas. Sin embargo, en estas se encontraron otros componentes del Pensamiento Histórico que no están contenidos en los modelos de Santisteban (2010) y Seixas y Morton (2013). Estos son: representaciones sociales, identidad, emociones, memoria, experiencia (dimensión personal) y preguntas históricas (dimensión metodológica).

Por ejemplo, la narrativa más compleja en la que se incluyen la experiencia, la identidad, la memoria y las emociones es el relato de Noa (A16_Zona Centro 1), quien explica la dictadura militar en Chile señalando que:

Se abordará un tema delicado: la represión, la tortura y la desaparición de muchos militantes y sujetos políticos en la época de la Unidad Popular (...) Este tema se tratará desde una perspectiva geográfica, con la categoría paisaje, ya que permite vincular los sucesos históricos con las emociones y sensaciones que aquel territorio o espacio produce. De esta manera las y los estudiantes lograrán situarse y entender cómo se sentían los ciudadanos durante esa época (...) Este punto se pretende abordar con material audiovisual proporcionado por el museo de DDHH [Derechos Humanos], por el sitio web Memoria Chilena y con la lectura de poemas realizados por los mismos detenidos en el Estadio Nacional.

La presencia de las representaciones sociales y como parte de ellas la experiencia, la identidad, la memoria y las emociones, conducen a la necesidad de incorporar, en el modelo, la dimensión personal del Pensamiento Histórico. Esta dimensión se relaciona con la idea de que el Pensamiento Histórico es contextual y es único, permitiendo abordar la historia desde una perspectiva local y decolonial.

- Segunda fase: consistió en sistematizar una propuesta para el desarrollo del Pensamiento Histórico que incluya los elementos que han sido identificados en las narrativas y que no están presentes en las categorías 
iniciales de análisis. En paralelo se procede a analizar los otros modelos precedentes de Pensamiento Histórico. Se elabora un mapeo conceptual y se discute su estructura junto con los directores de la tesis, el Dr. Joan Pagès y la Dra. Liliana Bravo.

- Tercera fase: observar clases de didáctica en centros universitarios. En la primera clase Catalina abordó la temporalidad y el cambio utilizando como referentes a Santisteban (2007) y Pagès y Santisteban (1999). Ella incluyó: el tiempo histórico (lineal y simultáneo), las relaciones temporales (conciencia histórica), los operadores temporales, el cambio y la continuidad, la memoria histórica, el tiempo vivido y la experiencia. La primera clase de Catalina aportó al modelo: i) la sistematización de indicadores del componente temporalidad y cambio. El ejemplo se aprecia en el siguiente diálogo:

Profesora Catalina: El tiempo es algo complejo, pero según lo que dice Santisteban (2007), en la escuela generalmente se enseña el tiempo (...) desde la cronología y la periodización (...)

Millaray (estudiante): leí que en la enseñanza de la historia había que resignificar el tiempo histórico, no solo considerando las relaciones tiempo-espacio, sino también estableciendo relaciones entre el presente, el pasado y el futuro. Se supone que a partir de esto se desarrolla la conciencia histórica (...)

Hernán (estudiante): la construcción del futuro debe hacerse con una visión prospectiva, pero también desde la ciudadanía. Para desarrollar una ciudadanía consciente.

En la segunda clase el elemento central fue la problematización de los contenidos históricos. La problematización es el componente clave para la articulación entre los diferentes componentes del Pensamiento Histórico. La segunda clase de Catalina aportó al modelo: i) la inclusión de la problematización histórica y ii) la articulación de los diferentes componentes del Pensamiento Histórico. Un ejemplo se observa cuando la docente proyecta las figuras 1 y 2. 


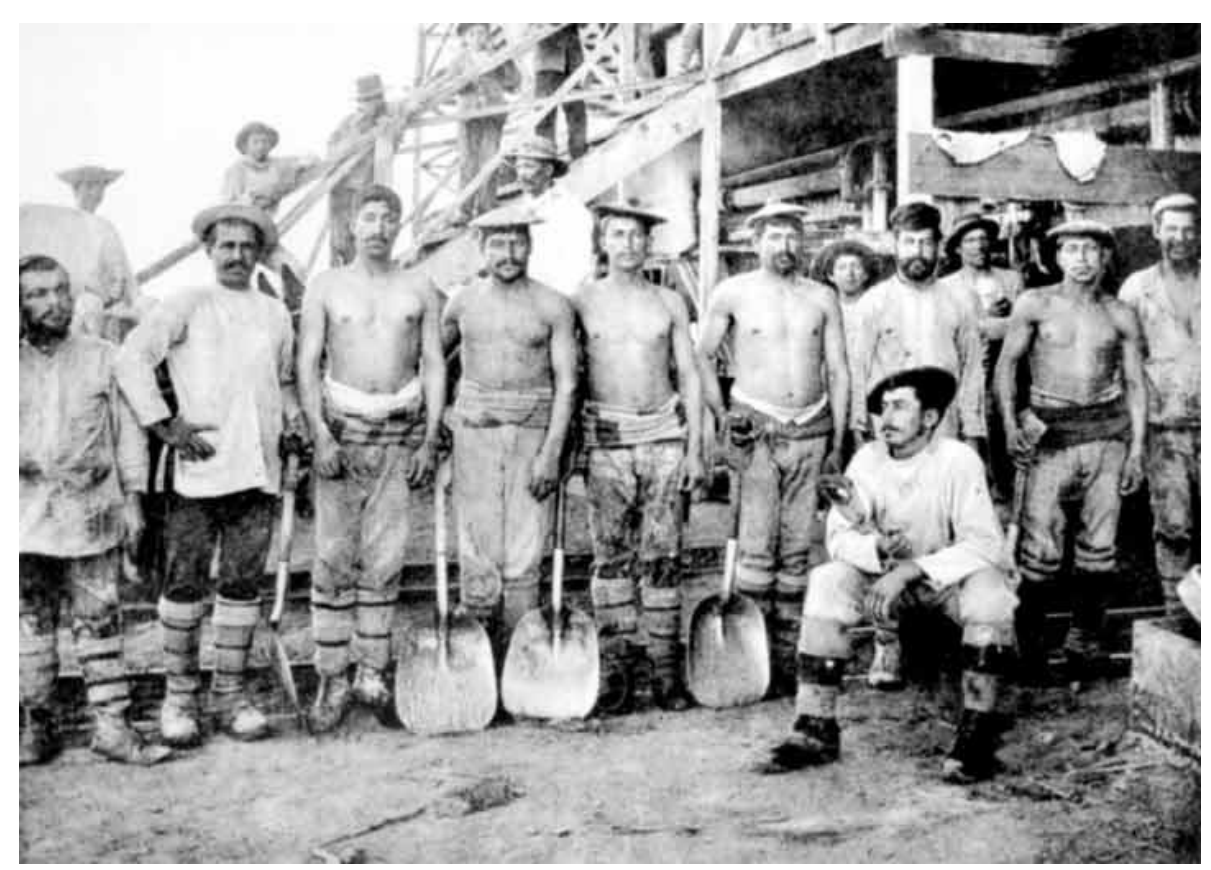

Figura 1.

Problematización histórica

Obreros del salitre en Chile a fines del siglo XIX.

https://centroderecursos.educarchile.cl/handle/20.500.12246/37673?show=full

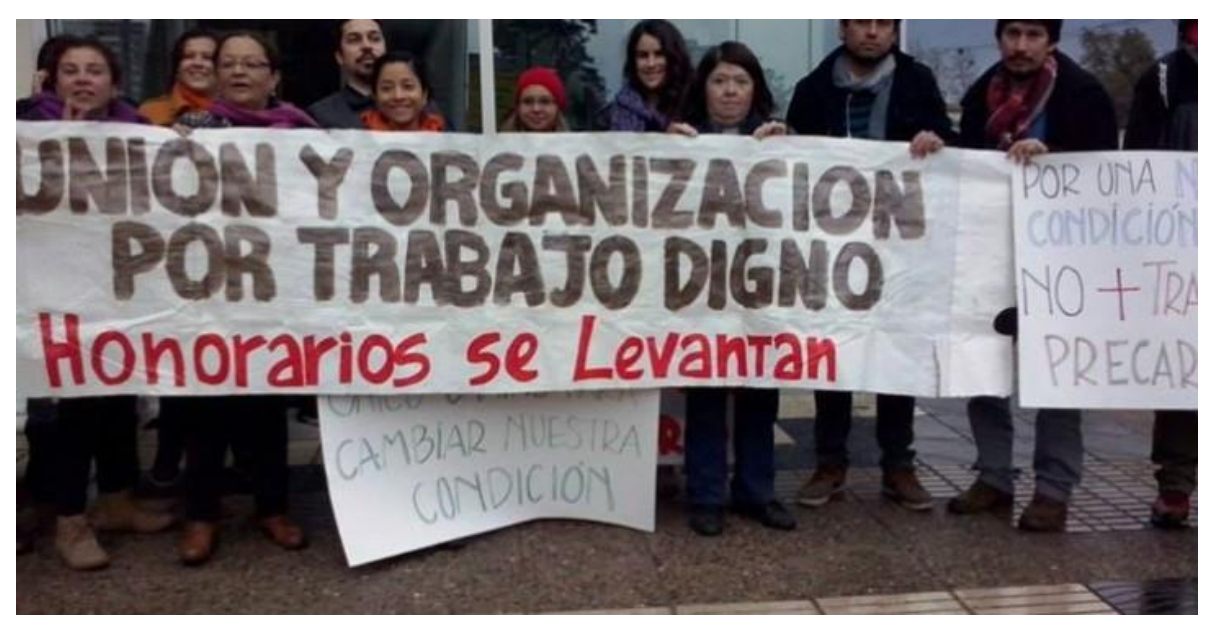

Figura 2.

Problematización histórica

Trabajadores sindicados en la actualidad.

https://www.elquintopoder.cl/trabajo/trabajadores-a-honorarios-vs-estado-de-chile/

La proyección de las imágenes permite que Catalina otorgue herramientas a sus estudiantes para que puedan desarrollar la problematización y articularla con otros componentes del Pensamiento Histórico:

Profesora Catalina: ¿A qué corresponde esta imagen? [izquierda]

Scarlet: La cuestión social y las condiciones obreras.

Profesora Catalina: Muy bien, ustedes están aportando conceptos. ¿Qué se propone a través de la imagen?

Millaray: (...) Se muestran condiciones indignas (...)

Profesora Catalina: Pero ¿A qué tipo de problema hacen alusión? ¿Qué ha cambiado y qué continúa? 
El ejemplo de la clase $\mathrm{N}^{\circ} 2$ de Catalina permite articular diferentes componentes del Pensamiento Histórico partiendo desde la problematización. Componentes como: utilización de fuentes, protagonistas históricos, temporalidad y cambio, perspectiva histórica y juicio ético.

En la tercera clase Catalina abordó los modelos de Pensamiento Histórico (Santisteban, 2010; Seixas y Morton, 2013; van Drie y van Boxtel, 2008), identificó sus componentes, y los ejemplifico generando vínculos con las experiencias de sus estudiantes en sus centros de práctica. Respecto a los modelos, ella señala que son "grandes categorías que permiten comprender lo que ocurrió y por consecuente estructurantes para cualquier análisis histórico (...), sirven para hacer comprensible y explicable la historia”. Para facilitar el proceso de organización de la enseñanza sugiere considerar cuatro etapas: I) explorar las representaciones sociales, II) introducir, modelar y confrontar conceptos (de primer orden), III) estructurar el conocimiento (conceptos de segundo orden) y IV) aplicar (conceptos de segundo orden)

La tercera clase de Catalina aportó al modelo: i) la importancia de articular las dimensiones y componentes del Pensamiento Histórico con el proceso de enseñanza y aprendizaje de la historia. Esto se incluye en la secuencia didáctica que se propone.

- Cuarta fase: Redefinir el mapeo conceptual, elaborar el modelo y los indicadores que se presentan en este artículo.

\subsection{Un modelo para el desarrollo del Pensamiento Histórico}

La estructura del modelo se traduce en dimensiones, componentes e indicadores. Se entiende por dimensión una categoría o ámbito del Pensamiento Histórico que agrupa diferentes componentes, comunes, que tributan a su desarrollo. Para construir cada una de las dimensiones se consideraron los siguientes procedimientos: fundamentación de la dimensión, incorporación de componentes, justificación, teorización, reconocimiento de los problemas de enseñanza y aprendizaje, y elaboración de indicadores. En la figura 3 se expone el modelo. 


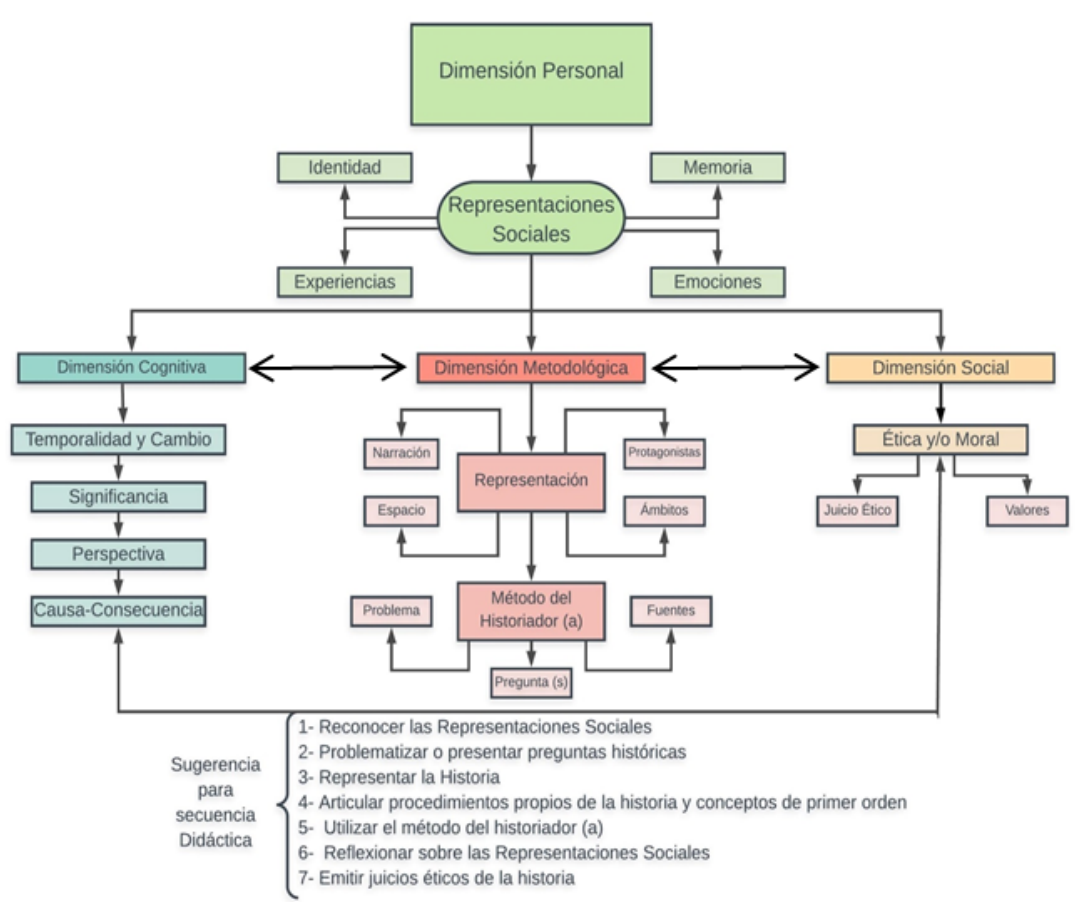

Figura 3.

Modelo para el desarrollo del Pensamiento Histórico

Chávez, 2020:79

\subsubsection{Dimensión Personal1}

Esta dimensión prologa el modelo porque el punto de partida de la enseñanza de la historia y del desarrollo del Pensamiento Histórico es el alumnado, niños, niñas o jóvenes que son seres históricos (Carr, 2017), que tienen historicidad y comprenden el mundo desde sus representaciones sociales. Esta dimensión también se relaciona con la conciencia histórica (Rüsen, 2004), puesto que se tiene en cuenta no solo a la persona en el presente, sino también se valora su pasado y se reflexiona sobre el futuro. La dimensión personal contribuye a comprender el Pensamiento Histórico desde la diversidad de elementos que forman parte de la enseñanza de la historia en las aulas, sin restringirlo a los procedimientos cognitivos de la historia (Plá, 2012). La comprensión de esta dimensión está vinculada a las particularidades del Pensamiento Histórico, es contextual, único, articulado y antinatural. Para incluirla en la enseñanza se exponen los indicadores en la Tabla 2. 
Tabla 2.

Indicadores

\begin{tabular}{|c|c|}
\hline \multirow[t]{5}{*}{$\begin{array}{l}\text { Dimensión } \\
\text { Personal }\end{array}$} & $\begin{array}{l}\text { Reconoce las representaciones sociales del alumnado en relación con } \\
\text { hechos, protagonistas o procesos históricos y las utiliza para la } \\
\text { construcción del conocimiento histórico (Jodelet, 1986; Pagès, 1996; } \\
\text { Carretero y Borrelli, 2012) }\end{array}$ \\
\hline & $\begin{array}{l}\text { Vincula el contenido histórico con elementos propios de la identidad de } \\
\text { los o las estudiantes (familiar, comunitario, cultural, ideológico o } \\
\text { nacional) (Carretero, 2007; Pagès, 2011; Carr, 2015 y 2017) }\end{array}$ \\
\hline & $\begin{array}{l}\text { Genera instancias para desarrollar o considerar experiencias históricas } \\
\text { a través de estrategias como testimonios, televisión, objetos, etc. (Carr, } \\
2017 \text {; Meneses et al., } 2019 \text { y 2020). }\end{array}$ \\
\hline & $\begin{array}{l}\text { Considera la memoria como parte de la construcción histórica, } \\
\text { desarrollando oportunidades para que los y las estudiantes utilicen la } \\
\text { memoria individual o colectiva (Pagès, 2008; Mattozzi, 2008; Pagès y } \\
\text { Santisteban, 2009) }\end{array}$ \\
\hline & $\begin{array}{l}\text { Incluye en la enseñanza de la historia las emociones de los estudiantes } \\
\text { o de los protagonistas históricos frente a procesos o hechos históricos } \\
\text { determinados (Latapi y Pagès, 2018; Castellví et al., 2019) }\end{array}$ \\
\hline
\end{tabular}

Chávez, 2020:8

\subsubsection{Dimensión Metodológica}

Esta dimensión pone en contacto al estudiantado con la historia y con los procedimientos de investigación científica. Es la clave que proporciona credibilidad y validez a la argumentación y explicación histórica, permitiendo que el estudiantado ponga en práctica los métodos del historiador(a) (Wineburg, 2001). Está conformada por la representación histórica y el método del historiador. Para comprender la dimensión hay que vincularla a algunas características del Pensamiento Histórico, por ejemplo, que es hipotético deductivo, articulado, reflexivo, crítico e interpretativo, progresivo y antinatural. La representación histórica mediatiza el contacto y el flujo de información entre la historia y las personas, "permite dar cuenta de cómo se transmite la historia, qué hechos y personajes forman parte de ella, y en qué escenarios se sitúa el relato" (Chávez y Pagès, 2019:777).

El método del historiador(a) son los procedimientos que se utilizan para conocer el pasado y reconstruirlo a través del uso de fuentes. Para ello, se propone abordar un tema socio-histórico desde la problematización (Bain, 2005; Dalongeville 2003) y la(s) pregunta(s) histórica(s) (Collingwood, 1952; Gruzinski, 2019). El problema y/o la pregunta han de contener, anticipando posibles respuestas, los elementos de otras dimensiones que se quieran desarrollar. Para incluirla se exponen los indicadores en la Tabla 3. 
Tabla 3.

Indicadores

\begin{tabular}{|c|c|}
\hline \multirow[t]{2}{*}{ Método del Historiador. } & $\begin{array}{l}\text { Problematiza y plantea preguntas históricas o genera instancias } \\
\text { para que el estudiantado por si mismo pueda problematizar o } \\
\text { proponer preguntas del contenido (Martineau, 1999; Dalongeville, } \\
\text { 2003; Bain, 2005; Hassani 2005; van Drie y van Boxtel, 2008; } \\
\text { Ford, 2014). }\end{array}$ \\
\hline & $\begin{array}{l}\text { Utiliza fuentes históricas y desarrolla estrategias que les permitan } \\
\text { a las y los estudiantes contextualizar, analizar y contrastar las } \\
\text { fuentes. (Santisteban, 2010; Seixas y Morton, 2013; Seixas et al. } \\
\text { 2015; Domínguez, 2015) }\end{array}$ \\
\hline \multirow[t]{3}{*}{$\begin{array}{l}\text { Representación } \\
\text { Histórica }\end{array}$} & $\begin{array}{l}\text { Representa la historia utilizando formas diversas o } \\
\text { complementarias, por ejemplo, la narrativa, la narrativa y los } \\
\text { medios audiovisuales o la narrativa y los testimonios (Chávez y } \\
\text { Pagès, 2019). }\end{array}$ \\
\hline & $\begin{array}{l}\text { Incluye en la representación histórica diferentes protagonistas } \\
\text { (Lautier, 1994; Santisteban, 2010; Plá, 2014; Pinochet, 2015; } \\
\text { Marolla, 2016) }\end{array}$ \\
\hline & $\begin{array}{l}\text { Considera en la representación histórica el espacio y la perspectiva } \\
\text { geohistórica (Hassani, 2005; Demattè et al., 2012; Mattozzi, 2014; } \\
\text { Borghi y Dondarini, 2019) }\end{array}$ \\
\hline
\end{tabular}

Chávez, 2020:97

\subsubsection{Dimensión Cognitiva}

Es la dimensión más indagada en la enseñanza de la historia y predomina en los modelos de Pensamiento Histórico de Hassani (2005); Santisteban et al. (2010); Seixas y Morton, (2013) y en la historiografía. La constituyen los procedimientos propios de la historia: temporalidad y cambio, significancia, perspectiva histórica, y causas y consecuencias.

La temporalidad y el cambio: la temporalidad la conforman el pasado, el presente y el futuro (Pagès, 1989) y las relaciones temporales bidireccionales o multidireccionales (Chávez y Pagès, 2020). El cambio, aparejado incondicionalmente a la continuidad, permite la comprensión y la organización de la temporalidad (Pagès y Santisteban, 1999). El tiempo histórico y los operadores temporales (Mattozzi, 1988) permiten representar el tiempo y organizar el relato. Para incluir la temporalidad y el cambio se exponen los indicadores en la tabla 4. 


\section{Tabla 4.}

Indicadores

Temporalidad y Cambio

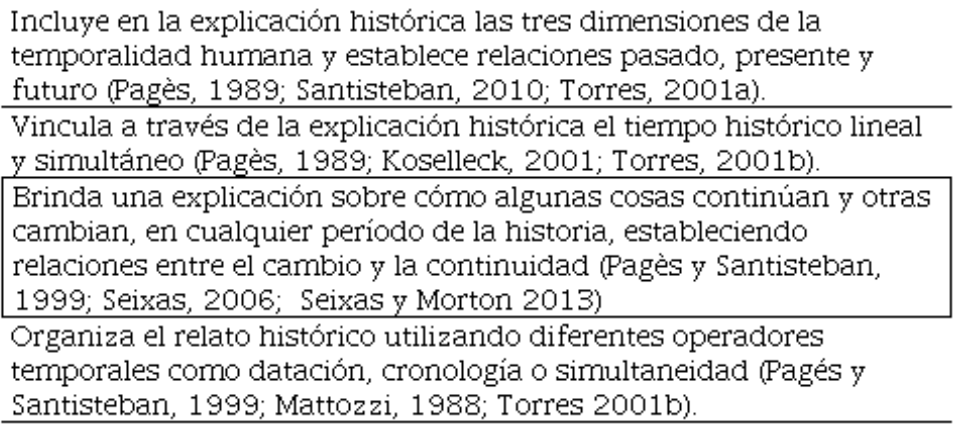

Chávez, 2020:107

La significancia histórica: consiste en seleccionar hechos, protagonistas o procesos que serán incluidos en la reconstrucción e interpretación del pasado (Collingwood, 1952; Ricoeur, 1990; Seixas y Peck, 2004; Seixas y Morton, 2013). Para incluir la significancia se exponen los indicadores en la Tabla 5.

\section{Tabla 5.}

Indicadores
Significancia Histórica

\begin{tabular}{|c|}
\hline $\begin{array}{l}\text { Explicita que algún o algunos de los elementos que incluye en su } \\
\text { explicación histórica es o son significativos porque provocaron } \\
\text { cambios o consecuencias en un periodo prolongado de tiempo } \\
\text { (Braudel, 2002; Seixas y Morton, 2013). }\end{array}$ \\
\hline $\begin{array}{l}\text { Considera en su explicación histórica la vinculación de un hecho, } \\
\text { proceso o elemento con diferentes ámbitos, por ejemplo, el político, } \\
\text { el económico, el social o el cultural. (Seixas y Morton, 2013). }\end{array}$ \\
\hline
\end{tabular}

Chávez, 2020:109

a perspectiva histórica: es el conocimiento y la comprensión interior de la historia (Collingwood, 1952) desde los sentimientos, experiencias, ideas o emociones de sus protagonistas (Seixas, 2006), tributa a la humanización de la historia, vinculándose a la empatía y a la contextualización. A la empatía histórica porque es posible desarrollar la acción cognitiva de ponerse en el pasado y pensar en una circunstancia histórica incluyendo valores, sentimientos o emociones (David, 2001). Y a la contextualización, porque es necesario pensar y pensarse en el tiempo (Wineburg, 2001). Para incluirla se exponen los indicadores en la tabla 6.

La perspectiva histórica: es el conocimiento y la comprensión interior de la historia (Collingwood, 1952) desde los sentimientos, experiencias, ideas o emociones de sus protagonistas (Seixas, 2006), tributa a la humanización de la historia, vinculándose a la empatía y a la contextualización. A la empatía histórica porque es posible desarrollar la acción cognitiva de ponerse en el pasado y pensar en una circunstancia histórica incluyendo valores, sentimientos o emociones (David, 2001). Y a la contextualización, porque es necesario pensar y pensarse 
en el tiempo (Wineburg, 2001). Para incluirla se exponen los indicadores en la tabla 6.

\section{Tabla 6.}

Indicadores

Perspectiva Histórica
Causas y Consecuencias
Incluye la perspectiva histórica de diferentes protagonistas (Seixas, 2006; Seixas y Morton, 2013; Seixas et al., 2015)

Vincula la perspectiva histórica con la utilización de fuentes (Seixas, 2006; Seixas y Morton, 2013; Seixas et al., 2015)

Desarrolla una perspectiva histórica contextualizada que permita comprender el actuar de personas en el pasado, sin caer en el presentismo (Wineburg, 2001; Seixas, 2006; Seixas y Morton, 2013)

Vincula la perspectiva histórica con la empatía (David, 2001; Lee y Ashby, 2001; Barton y Levstik, 2004).

Chávez, 2020:112

Causas y consecuencias: todo lo que ocurre es precedido por causas que provocan consecuencias. La explicación causal: i) se basa en identificar los porqué (Heller, 1982), ii) está relacionada con la temporalidad, especialmente, con el cambio (Domínguez, 2015) y con las duraciones, por ejemplo, causas de larga o corta duración (Chapman, 2015), iii) requiere considerar múltiples causas y consecuencias, aunque no todas poseen la misma jerarquía en cuanto a la concurrencia de un hecho o proceso (Seixas y Morton, 2013). Para incluirlas se exponen los indicadores en la Tabla 7.

\section{Tabla 7.}

Indicadores

Incluye en su explicación histórica causas y consecuencias (Seixas, 2006 y Seixas y Morton, 2013)

Analiza los hechos o procesos históricos dando cuenta de la multicausalidad y la jerarquía causal (Seixas, 2006; Hassani, 2005; Seixas y Morton, 2013)

Establece relaciones entre las diferentes causas que explican un hecho o proceso histórico (Carr, 1978; Seixas y Morton, 2013;

Dominguez, 2015).

Analiza los hechos o procesos históricos dando cuenta de las múltiples consecuencias y la jerarquía de consecuencias (Le Goff, 1992; Seixas, 2006; Seixas y Morton, 2013)

Establece relaciones causa, consecuencia, cambio y lo continuidad (Lautier, 1994; Torres, 2001b; Stearns, 2004; Duquette, 2011; Seixas y Morton, 2013)

Chávez, 2020:115

\subsubsection{Dimensión Social}

La Dimensión incluye el juicio y los valores éticos y/o morales. Se fundamenta en que la historia ha de servir para la vida y estar al servicio de la sociedad, permitiendo un diálogo constante desde el presente, con el pasado, y proyectando el futuro (Santisteban y Anguera, 2014). La dimensión social es un punto de encuentro entre el Pensamiento Histórico y la Conciencia Histórica (Rüsen, 
2004). Los juicios éticos y/o morales son reflexiones argumentadas respecto a situaciones que ocurrieron en el pasado [que son reprochables desde el presente e irrepetibles para el futuro] y que fueron conflictivas desde el punto de vista ético y/o moral (Bermejo, 1991). Situaciones como las guerras mundiales o las misiones de indígenas en América (http://historicalthinking.ca/historicalsignificance). Los valores que se implican con los juicios éticos y/o morales son característicos de un período particular (Le Goff, 2014). Para incluir la dimensión social se exponen los indicadores en la tabla 8.

\section{Tabla 8.}

Indicadores

Juicios Ético y lo Morales
Incorpora y problematiza algún tema moral y jo ético, asociado al proceso y lo acontecimiento histórico que relata (Seixas, 2006; Seixas y Morton, 2013)

Reconoce las diferencias éticas y morales del contexto histórico en que operan y la actualidad (contextualización) (Wineburg, 2001 y Seixas, 2006)

Vincula el juicio ético y lo moral a valores democráticos como la justicia, la igualdad, la libertad, la verdad, la tolerancia, el respecto de los DDHH (Orlandi, 1975; Carr, 1978; Heller, 1982; Todorov, 1993; Gadamer, 1993; Rüsen, 2007)

Vincula juicio ético y lo moral con el uso de fuentes y evidencias y 10 perspectiva histórica (Seixas, 2006; Seixas y Morton, 2013; Seixas et al., 2015)

Genera instancias para que las y los estudiantes por sí mismos puedan emitir juicios morales (Seixas, 2006)

Chávez, 2020:120

\section{Conclusiones}

La sistematización de un modelo hispanoparlante pensado desde la enseñanza y para el desarrollo del Pensamiento Histórico es un aporte para la didáctica, para la formación inicial docente y para la enseñanza de la disciplina porque:

- Permite comprender la articulación de las dimensiones y componentes que constituyen el Pensamiento Histórico y sugiere indicadores para guiar la enseñanza de la historia. Esto contribuye a subsanar las dificultades que tiene el profesorado para integrar estos elementos como lo señalan Barton y Levstik (2004); Chávez (2020); Ramírez y Pagès (2019). La articulación de las dimensiones y componentes se expone a través de la teorización de las características del Pensamiento Histórico, es decir, que es articulado y jerarquizable y, además, en el ejemplo de la clase $\mathrm{N}^{\circ} 2$ y No3 de Catalina. En la clase No2 ella comienza a desarrollar el Pensamiento Histórico a través de la problematización (dimensión metodológica) y posteriormente articula este componente con el uso de fuentes, los y las protagonistas históricas, el cambio y la continuidad, la perspectiva histórica y el juicio ético. En la clase No 3 expone cuatro etapas que permiten desarrollar la articulación y la jerarquización, estas etapas son: I) explorar las representaciones sociales, II) introducir, modelar y confrontar conceptos (de primer orden), III) 
estructurar el conocimiento (conceptos de segundo orden) y IV) aplicar (conceptos de segundo orden).

- Contribuye a entender que el Pensamiento Histórico es un proceso en el que se entreteje lo individual y lo social. Por esto, el modelo pone de manifiesto la importancia de la dimensión personal del Pensamiento Histórico cuyo punto de partida es el ser histórico. En este sentido el ejemplo de la narrativa de Noa en la que se incluyen la experiencia, la identidad, la memoria y las emociones es un ejemplo concreto sobre cómo abordar esta dimensión en la enseñanza de la historia.

- Concibe al Pensamiento Histórico como un proceso dinámico y transformador, pues implica un flujo constante de información y reflexión, cuya finalidad es resignificar la forma natural en que el ser histórico comprende su realidad. Esto queda de manifiesto en la secuencia didáctica que se sugiere desde el modelo de Pensamiento Histórico propuesto y en la articulación de las dimensiones y componentes expuesta en las clases No 2 y No3 de Catalina.

También cabe concluir que el modelo expuesto en este artículo difiere de sus precedentes porque: i) ha sido pensado desde la enseñanza y creado a partir de la interacción de la teoría y el trabajo de campo, esto ha permitido visibilizar la dimensión personal que no había sido expuesta en los modelos precedentes; ii) en su estructura, pensada para promover la articulación, explicita dimensiones, componentes e indicadores, por tanto, facilita la comprensión del complejo entramado de lo que significa pensar históricamente y también contribuye a traducción pedagógica del lenguaje historiográfico; iii) incluye y valora la dimensión personal cuyo punto de partida es el ser histórico, esta dimensión y sus componentes permiten que la enseñanza de la historia adopte un enfoque decolonial y una perspectiva de género al visibilizar y otorgar relevancia a las representaciones sociales, por ello se piensa que es una propuesta que contribuye a la necesidad detectada por Chaparro et al., (2020); iv) detalla la metodología utilizada para su construcción, en ella se consideran cuatro fases en las que interactúa la teoría y la información proveniente de narrativas y observación de clases de didáctica y $\mathbf{v}$ ) responde a la realidad cultural chilena e iberoamericana dado que el levantamiento de información empírica se ha llevado a cabo en Chile y teóricamente se han considerado investigaciones de autores iberoamericanos entre ellos Domínguez (2015); Pagès (1989); Pagès y Santisteban (1999); Plá (2012 y 2014); Santisteban (2010); Santisteban et al. (2010).

Finalmente, señalar que el modelo representa una actualización complementaria, y por supuesto abierta a la reflexión, al modelo hispanoparlante de Santisteban (2010) y Santisteban et al. (2010) y a los modelos precedentes de Duquette (2011); Hassani (2005); Martineau (1999) y Seixas y Morton (2013).

\section{Referencias}

Arnal, J.; Rincón, D. y Latorre, A. (1992). La investigación educativa. Fundamentos y metodologias. Labor.

Bain, R. (2005). ¿Ellos pensaban que la tierra era plana? Aplicación de los principios de Cómo aprende la gente, en la enseñanza de la Historia en la Educación Secundaria. En Donovan, S. y Bransford, J. (Eds). How Students Learn: History, Mathematics, 
and Science in the Classroom (2-24). Academia Nacional de Ciencias de Estados Unidos.

Bardin, L. (2002). Análisis de Contenido. Akal.

Barton, K.; Levstik, L. (2004). Teaching History for the common good. Lawrence Erlbaum Associates.

Belanger, E. (2011). How Now? Historical Thinking, Reflective Teaching, and the Next Generation of History Teachers. The Journal of American History, 97(4), 1079-1088.

Bermejo, J. (1991). Fundamentación Lógica de la Historia. AKAL Universitaria.

Bisquerra, R. (2009) (coord.). Metodología de la investigación educativa. La muralla.

Borghi, B.; Dondarini, R. (2019). Un Manifesto per la Didattica della Storia. Didattica della storia-Journal of Research and Didactics of History, 1, 1-20.

Braudel, F. (2002). La Historia en busca del mundo. Crítica.

Carr, D. (2015). Tiempo, narrativa e historia. Prometeo.

Carr, D. (2017). Experiencia e historia. Prometeo.

Carr, E. (1978). ¿Qué es la historia? Seix Barral.

Carretero, M. y Borrelli, M. (2012). La historia reciente en la escuela: propuestas para pensar históricamente. En Carretero, M. y Castorina, J. La construcción del conocimiento histórico. Enseñanza, narración e identidades (103-129). Paidós.

Carretero, M.; Castorina, J. (2012). La construcción del conocimiento histórico. Enseñanza, narración e identidades. Paidós.

Carretero, M.; Kriger, M. (2012). Enseñanza de la historia e identidad nacional a través de las efemérides escolares. En Carretero, M. y Castorina, J. (2012). La construcción del conocimiento histórico. Enseñanza, narración e identidades (54-80). Paidós.

Carretero, M. (2007). Documentos de identidad, la construcción de la memoria histórica en un mundo global. Paidós- Entornos.

Castellví, J., Massip, M.; Pagès, J. (2019). Emociones y pensamiento crítico en la era digital: un estudio con alumnado de formación inicial. REIDICS: Revista de Investigación en Didáctica de las Ciencias Sociales, 5, 23-41.

Chaparro, A.; Felices, M. M.; Triviño, L. (2020). La investigación en pensamiento histórico. Un estudio a través de las tesis doctorales de Ciencias Sociales (1995-2020). Panta Rei: revista digital de Historia y didáctica de la Historia, 93-147.

Chapman, A. (2015). Developing historical and metahistorical thinking in history classrooms: reflections on research and practice in England. Diálogos, 19, 9-55.

Chávez, C. (2020). Formación del Pensamiento Histórico en estudiantes de formación inicial docente. Un estudio de casos en universidades chilenas. (Tesis Doctoral). Universidad Autónoma de Barcelona.

Chávez, C.; Pagès, J. (2019). Habilidades de pensamiento histórico desarrolladas por estudiantes de formación inicial del profesorado de historia de Chile. En Hortas, M. J., Dias, A. D’Almeida y De-Alba-Fernández, N. (2019). Enseñar y aprender didáctica de las ciencias sociales: la formación del profesorado desde una perspectiva sociocritica. Simposio en Didáctica de las Ciencias Sociales (776-788). Lisboa: AUPDCS-ESELX.

Chávez, C.; Pagès, J. (2020). Habilidades de conciencia histórica temporal en estudiantes de Formación Inicial de Chile. REIDICS, 6, 24-42.

Collingwood, R. (1952). La idea de la historia. Fondo de Cultura Económica. 
Dalongeville, A. (2003). Noción y práctica de la situación-problema en historia. Enseñanza de las Ciencias Sociales, 2, 3-12.

David, O. (2001). In Pursiuit of Historical Empathy. In David, O.; Yaeguer, E. y Foster, S. (2001) (Eds). Historical Empathy and Perspective Taking in the Social Studies (1-12). Rowman \& Littlefield Publishers.

Demattè, F.; Lotti, P.; Perillo, E.; Rabuiti, S. (2012). La geostoria alla prova dei manuali. Proposte di unità di apprendimento per il biennio delle scuole superiori. II Bollettino di Clio. Maggio, XII, 15-24.

Domínguez, J. (2015). Pensamiento histórico y evaluación de competencias. Graó.

Duquette, C. (2011). Le rapport entre la pensee historique et la conscience historique. Elaboration d'un modèle d'interaction lors de l'apprentissage de l'histoire chez les élèves de cinquième secondaire des écoles francophones du Québec. (Tesis doctoral). Université Laval, Québec.

Flick, U. (2015). El diseño de investigación cualitativa. Morata.

Ford, A. (2014). Setting us free? Building meaningful models of progression for a 'postlevels' world. Teaching History. 157, 28-41.

Freire, P. (2004). Pedagogía para la liberación. Graó.

Gadamer, H. (1993). El problema de la conciencia histórica. Tecnos.

Gibson, L.; Peck, C. (2020). More than a Methods Course: Teaching Preservice Teachers to Think Historically. In C. Berg y T. Christou (Eds.), Palgrave handbook of history and social studies education (213-251). Palgrave Macmillan.

Gómez, C. J.; Ortuño, J.; Molina, S. (2014). Aprender a pensar históricamente. Retos para la historia en el siglo XXI. Tempo e Argumento, 6(11), 5-27.

Gruzinski, S. (2019). ¿Para qué sirve la historia? Alianza Editorial.

Hassani, I. (2005). Pensée historienne et apprentissage de l' 'historie. L' Harmattan.

Heller, A. (1982). Teoría de la Historia. Fontamara.

Jodelet, D. (1986). La representación social: fenómenos, concepto y teoría. En Moscovici, S. (comp.). Psicología Social II (469-494). Paidós.

Koselleck, R. (2001). Los estratos del tiempo: estudios sobre la historia. Paidós.

Latapí, P.; Pagès, J. (2018). Debates en la historiografía y en las investigaciones sobre enseñanza de la historia en torno a las cogniciones y a las emociones. Clío \& Asociados. La historia enseñada, 27, 108-117.

Lautier, N. (1994). La compréhension de l'histoire: un modèle spécifique. Revue française de pédagogie, 106, 66-77.

Le Goff, J. (1992). Recerca e insegnamento della storia. ILI.

Le Goff, J. (2014). ¿Realmente es necesario cortar la historia en rebanadas? Fondo de Cultura Económica.

Lee, P.; Ashby, R. (2001). Empathy, perspective taking, and rational understanding. In David, O.; Yaeguer, E. y Foster, S. (2001) (eds). Historical Empathy and Perspective Taking in the Social Studies (21-50). Rowman \& Littlefield Publishers.

Lévesque, S. (2008). Thinking historically: Educating students for the twenty-first century. University of Toronto Press.

Marolla, J. (2016). La inclusión de las mujeres en la enseñanza de la historia y las ciencias sociales. Estudio colectivo en las aulas chilenas sobre sus posibilidades y limitaciones (tesis doctoral). Universitat Autònoma de Barcelona.

Martineau, R. (1999). L'Historie á lècole. Matière à penser... L'Harmattan. 
Mattozzi, I. (1988). I bambini, il tempo, la storia: educazione temporale e curricolo di storia nella scuola elementare, en CIDI di Firenze (a cura di). Tempo e spazio, dimensioni del sapere. Dalle ipotesi teoriche alle practiche didattiche, 65- 81.

Mattozzi, I. (2008). Memoria y formación histórica, la memoria en la clase de Historia. Íber,Didáctica de las Ciencias Sociales, Historia y Geografía, 55, 30-42.

Mattozzi, I. (2014). ¿Quién tiene miedo de la Geohistoria? Enseñanza de las ciencias sociales, 13, 85-105.

Maxwell, J. (2005). Diseño de investigación cualitativa. Un enfoque interactivo. Gedisa.

Meneses, B.; González, N.; Santisteban, A. (2019). Aprender desde la experiencia histórica. Representaciones del profesorado sobre el uso de la historia oral como estrategia didáctica. El Futuro del Pasado, 10, 257-286.

Meneses, B.; González, N.; Santisteban, A. (2020). La experiencia histórica del alumnado y la historia oral en la enseñanza. Historia y Memoria, 20, 309-343.

Orlandi, L. (1975). Evaluación del aprendizaje en estudios sociales de la escuela secundaria. En Bloom, B.; Hastings, J. T. y Madaus, G.F. Evaluación del aprendizaje, volumen 2 (243-315). Troquel.

Pagès, J.; Santisteban, A. (1999) La enseñanza del tiempo histórico: una propuesta para superar viejos problemas. En García, T. (coord.). Curriculum de las ciencias sociales para el siglo XXI, que contenidos y para qué(187-207). Díada.

Pagès, J.; Santisteban, A. (2009). Cambios y continuidades, aprender la temporalidad histórica. En Guimarães, S. (org.). Ensino fundamental, conteúdos, metodologias e prácticas (197-240). Alínea.

Pagés, J. (1989). Aproximación a un currículum sobre el tiempo histórico. En Rodríguez, J. Enseñar Historia. Nuevas propuestas (107-138). Cuadernos de Pedagogía. Laia.

Pagés, J. (1996). Las representaciones de los estudiantes de maestro sobre las Ciencias Sociales: ¿cuáles son?, ¿cómo aprovecharlas? Investigación en la Escuela, 28, 103-114.

Pagès, J. (2008). El lugar de la memoria en la enseñanza de la historia. Íber, Didáctica de las Ciencias Sociales, Historia y Geografía, 55, 43-53.

Pagès, J. (2011) Las reflexiones entre investigación y práctica docente en la enseñanza de la historia. En López, R.; Velasco, L.; Santidrián, V. y Armas, X. (ed.). Pensar históricamente en tiempos de globalización. Universidad de Santiago de Compostela.

Pagès, J.; Santisteban, A. (2011). Enseñar y aprender ciencias sociales. En Santisteban, A. y Pagès, J. (coord.). Didáctica del conocimiento del medio social y cultural en la educación primaria. Ciencias sociales para aprender, pensar y actuar (23-39). Síntesis.

Pinochet, S. (2015). Profesor, profesora ¿por qué los niños y las niñas no aparecen en la historia? Concepciones del profesorado y el alumnado sobre la historia de la infancia (tesis doctoral). Universitat Autònoma de Barcelona.

Plá, S. (2012). La enseñanza de la historia como objeto de investigación. Secuencia. Revista de historia y ciencias sociales, 84, 161-184.

Plá, S. (2014). Ciudadanía y competitividad en la enseñanza de la historia. Los casos de México, Argentina y Uruguay. Universidad Iberoamericana.

Ramírez Achoy, J.; Pagès, J. (2019). Pensar históricamente en la universidad: ¿̨cómo aprende historia el futuro profesorado de estudios sociales en Costa Rica? En Hortas, M. J.; Dias, A. D’Almeida y De-Alba-Fernández, N. (2019). Enseñar y aprender didáctica de las ciencias sociales: la formación del profesorado desde una perspectiva sociocritica (789-798). Lisboa: AUPDCS-ESELX. 
Reis, P.; Climent, N. (coord.) (2012). Narrativas de profesores: reflexiones en torno al desarrollo personal y profesional. Universidad Internacional de Andalucía.

Ricoeur, P. (1990). Historia y Verdad. Encuentro.

Ricoeur, P. (2000). La memoria, la historia, el olvido. Fondo de Cultura Económica.

Rojas, R. (2015). Aspectos teóricos sobre el proceso de formación de investigadores sociales. En Abero, L.; Berardi, L.; Capocasale, A.; García, S. y Rojas, R. Investigación Educativa. CLACSO.

Ruíz, J. (2012). Metodología de la Investigación cualitativa. Universidad de Deusto.

Rüsen, J. (2004). Historical Consciousness: Narrative Structure, Moral Function, and Ontogenetic Development. In Seixas, P. (eds.) (2004). Theorizing Historical Consciousness (63-85). University of Toronto Press.

Rüsen, J. (2007). Memory, history and the quest for the future. En Cajani, L. y Ross, A. (ed.). History Teaching, Identities and Citizenship. European Issues in Children's Identity and Citizenship 7 (13-34). Trentham Books.

Sáiz, J.; Gómez, C. (2016). Investigar el pensamiento histórico y narrativo en la formación del profesorado: fundamentos teóricos y metodológicos. Revista Electrónica Interuniversitaria de Formación del Profesorado, 19(1), 175-190.

Sandoval, C. (1996). Investigación Cualitativa. Instituto colombiano para el fomento de la Educación Superior.

Sant, E.; Pagés, J.; Santisteban, A.; González, N.; Oller, M. (2014). ¿Cómo podemos analizar la competencia narrativa del alumnado en el aprendizaje de la Historia? Clío \& Asociados. La historia enseñada, 18-19, 166-182.

Santisteban, A (2010). La formación de competencias de pensamiento histórico. Clío \& Asociados. La historia enseñada, 14, 34-56.

Santisteban, A.; Anguera, C. (2014). Formación de la conciencia histórica y educación para el futuro. Clio \& Asociados. La historia enseñada, 18-19, 249-267.

Santisteban, A.; González, N.; Pagès, J. (2010). Una investigación sobre la formación del pensamiento histórico. En Ávila, R.; Rivero, P y Domínguez, P. (coords.). Metodología de investigación en Didáctica de las Ciencias Sociales (115-128). Fernando el Católico -Diputación de Zaragoza/ AUPDCS.

Santisteban, A. (2007) Una investigación sobre cómo se aprende a enseñar el tiempo histórico. Enseñanza de las Ciencias Sociales, 6, 19-29.

Seixas, P. (2006) Benchmarks of historical thinking: a framework for assessment in Canada. En Seixas, P. (2008). 'Scaling Up' the Benchmarks of Historical Thinking $A$ Report on the Vancouver. Meetings, February 14-15. University of British Columbia.

Seixas, P.; Morton, T. (2013). The big six: Historical thinking concepts. Nelson Education.

Seixas, P.; Peck, C. (2004). Teaching historical thinking. In Sears, A. y Wright, I. (eds.). Challengens and Prospects for Canadian Social Studies (109-117). Pacific Educational Press.

Seixas, P., Gibson, L.; Ercikan, K. (2015). A design process for assessing historical thinking. The Case of One-Hour Test. En Seixas, P. y Ericikan, K. (eds). New Directions in Assessing Historical Thinking (102-117). Routledge.

Stearns, P. (2004). Thinking History. American Historical Association.

Todorov, T. (1993). Las morales de la historia. Paidós.

Torres, P. (2001a). La enseñanza del tiempo histórico. Proyecto didáctico Quirón. De la Torre. 
Torres, P. (2001b).Didáctica de la historia y educación de la temporalidad: Tiempo social y tiempo histórico. Universidad Nacional de Educación a Distancia.

Van Drie, J. y Van Boxtel, C. (2008). Historical reasoning: towards a framework for analyzing students' reasoning about the past. Educational Psychol, 20(2), 87-110.

Wineburg, S. (1991). Historical problem solving: a study of the cognitive processes used in the evaluation of documentary and pictorial evidence. Journal of Educational Psychology, 83, 73-87.

Wineburg, S. (2001). Historical thinking and other unnaturals acts: charting the future of teaching the past. Temple University Press.

\section{Notas}

1 Para la descripción en extenso del modelo consultar Chávez (2020). 